\title{
On constrained stochastic controllability of dynamical systems with multiple delays in control
}

\author{
B. SIKORA ${ }^{1 *}$ and J. KLAMKA ${ }^{2}$ \\ ${ }^{1}$ Silesian University of Technology, 23 Kaszubska St., 44-100 Gliwice, Poland \\ ${ }^{2}$ Silesian University of Technology, 16 Akademicka St., 44-100 Gliwice, Poland
}

\begin{abstract}
Linear, continuous stochastic dynamical systems with multiple delays in control are studied in the paper. Their relative stochastic controllability with constrained control is discussed. The definitions of various type of constrained relative and absolute stochastic controllability for linear systems with delays in control are introduced. Criteria of relative and absolute stochastic controllability with constrained control are established. Constraints on control values are considered. Mutual implications between constrained relative stochastic controllability of systems with and without delays are studied as well as implications between constrained relative and absolute stochastic controllability of systems with delay in control.
\end{abstract}

Key words: controllability, multiple delays in control, constrained stochastic controllability, supporting function.

\section{Introduction}

Roughly speaking, controllability generally means, that it is possible to steer a dynamical control system from an arbitrary initial state to an arbitrary final state using the set of admissible controls. It should be mentioned, that in the literature there are many different definitions of controllability, which strongly depend, on one hand, on a class of dynamical control systems and on the other hand, on the form of admissible controls.

Control theory is an interdisciplinary branch of engineering and mathematics that deals with influence behavior of dynamical systems. Controllability is one of the fundamental concepts in mathematical control theory. This is a qualitative property of dynamical control systems and is of particular importance in control theory. Systematic study of controllability was started at the beginning of sixties in the last century, when the theory of controllability based on the description in the form of state space for both time-invariant and time-varying linear control systems was worked out.

In recent years various controllability problems for different types of linear semilinear and nonlinear dynamical systems have been considered in many publications and monographs [1]. However, it should be stressed, that the most literature in this direction has been mainly concerned with different controllability problems for dynamical systems with unconstrained controls and without delays in the state variables or in the controls. Moreover, it should be mentioned, that only a few papers $[2,3]$ have been devoted to a systematic study of relative or complete controllability problems for stochastic dynamical systems with different types of delays in controls.

As was stated above controllability of dynamical systems is one of the main elements in their analysis. For the sake of the abundance of mathematical models of dynamical systems with delays, the controllability problem for such systems is especially important. Controllability problems for various types of linear dynamical systems have been considered in may publications (see the survey [4]). Controllability of both deterministic $[1,5]$ and stochastic $([6,7]$ linear systems with delays, and stochastic systems without delays [8-11] are studied. However, most literature in this field has been concerned with unconstrained controls. Some criteria for constrained controllability of linear dynamical systems with delays in control are presented in [12] and [13].

Stochastic observability is the concept dual to stochastic controllability. Stochastic observability has been considered, among others, in [14-16].

Linear stochastic ordinary differential equations containing the Wiener process have application, among others, in economic processes modeling $[17,18]$.

In the present paper, linear stochastic dynamical systems with multiple, time-dependent delays in control are studied. Constraints on control values are considered. The aim of the paper is to formulate criteria of stochastic relative controllability with constrained control.

\section{Mathematical model}

In the paper we use the following standard notation introduced by Zabczyk in [8]. Let $(\Omega, F, P)$ be a complete probability space with the probability measure $P$ defined on a set of events $\Omega$ and a filtration $\left\{F_{t}: t \in\langle 0, T\rangle\right\}$ generated by an $n$-dimensional Wiener process $\{w(s): 0 \leq s \leq t\}$ defined on $(\Omega, F, P)$. Let $L^{2}\left(\Omega, F_{t}, \mathbf{R}^{n}\right)$ denote the Hilbert space of all $F_{t}$-measurable square integrable random variables with values in $\mathbf{R}^{n}$ and let $L_{F}^{2}\left(\langle 0, T\rangle, \mathbf{R}^{n}\right)$ denote the Hilbert space of all square integrable and $F_{t}$-measurable processes with val-

*e-mail: beata.sikora@polsl.pl 
B. Sikora and J. Klamka

ues in $\mathbf{R}^{n}$. We consider linear, continuous, finite-dimensional dynamical systems with time-dependent multiple delays in control described by the following ordinary differential state equation

$$
\begin{aligned}
d x(t)= & \left(A(t) x(t)+\sum_{i=0}^{M} B_{i}(t) u\left(v_{i}(t)\right)\right) d t \\
& +\sigma(t) d w(t), \quad t \in\langle 0, T\rangle,
\end{aligned}
$$

where $x(t) \in \mathbf{R}^{n}$ - the instantaneous state $n$-vector, $u \in$ $L_{\text {loc }}^{2}\left(\langle 0, T\rangle, \mathbf{R}^{m}\right)$ - the control, $A(t)-n \times n$ dimensional matrix with elements, $a_{k j} \in L_{\text {loc }}^{1}(\langle 0, T\rangle, \mathbf{R}), k, j=1, \ldots, n$, $B_{i}(t)(i=0,1, \ldots, M)-n \times m$ dimensional matrices with elements, $b_{i k j} \in L_{\text {loc }}^{2}(\langle 0, T\rangle, \mathbf{R}), k=1, \ldots, n, j=1, \ldots, m$, $v_{i}:\langle 0, T\rangle \rightarrow \mathbf{R}, i=0,1, \ldots, M-$ absolutely continuous, strictly increasing functions, satisfying $v_{M}(t)<v_{M-1}<$ $\ldots<v_{k}(t)<\ldots<v_{1}(t)<v_{0}(t)=t, t \in\langle 0, T\rangle$, where $v_{i}(t)=t-h_{i}(t)$ and $h_{i}(t) \geq 0, i=0,1, \ldots, M$ are time dependent delays in control, $\sigma(t)-n \times n$ dimensional matrix with continuous elements $\sigma_{k j}, k, j=1, \ldots, n, w(t)(t \geq 0)-$ $n$-dimensional Wiener process, i.e. a stochastic, process characterized by three properties: $w(0)=0$ almost surely, $w(t)$ has independent increment with $w(t)-w(s) \sim \mathcal{N}(0, t-s)$ for $0 \leq s \leq t$, the function $t \mapsto w(t)$ is almost surely continuous.

Let $U \subset \mathbf{R}^{m}$ be any non-empty set. The set $U_{a d}=$ $L_{F}^{2}(\langle 0, T\rangle, U)$ of square integrable functions in $\langle 0, T\rangle$ with values in $U$ is the set of admissible controls for the dynamical system (1). The initial condition $z(0)=\left\{x_{0}, u_{0}\right\} \in \mathbf{R}^{n} \times$ $L_{F}^{2}\left(\left\langle v_{M}(0), 0\right\rangle, U\right)$, where $x_{0}=x(0) \in \mathbf{R}^{n}$, and $u_{0}$ is the given initial function in $\left\langle v_{M}(0), 0\right\rangle$, is called the initial complete state of the dynamical system (1). For the given initial condition $z(0)$ and an admissible control $u \in L_{F}^{2}(\langle 0, T\rangle, U)$, for every $t \in\langle 0, T\rangle$ there exists a unique, absolutely continuous solution $x(t, z(0), u) \in L^{2}\left(\Omega, F_{t}, \mathbf{R}^{n}\right)$ of the linear stochastic differential state equation (1). This solution has the form

$$
\begin{gathered}
x(t, z(0), u)=\Phi(t, 0) x_{0} \\
+\int_{0}^{t} \Phi(t, s) \sum_{i=0}^{M} B_{i}(s) u\left(v_{i}(s)\right) d s \\
+\int_{0}^{t} \Phi(t, s) \sigma(s) d w(s),
\end{gathered}
$$

where $\Phi(t, s)$ is $n \times n$ dimensional transition matrix of the linear system

$$
d x(t)=A(t) x(t) d t .
$$

In the case of a dynamical system with delays, only a complete state

$$
z(t)=\left(x(t), u_{t}(\tau)\right),
$$

where $u_{t}(\tau)=u(\tau)$ for $\tau \in\left\langle v_{M}(t), t\right)$, fully describes the behavior of the dynamical system at time $t$.

A special case of the system (1) is the linear, stationary, finite-dimensional dynamical systems with constant multiple delays in control described by the following ordinary differential state equation

$$
d x(t)=\left(A x(t)+\sum_{i=0}^{M} B_{i} u\left(t-h_{i}\right)\right) d t+\sigma d w(t),
$$

where $A-n \times n$ dimensional matrix with elements, $a_{k j} \in$ $\mathbf{R}, k, j=1,2, \ldots, n, B_{i}(i=0,1, \ldots, M)-(n \times m)$ dimensional matrices with elements, $b_{i k j} \in \mathbf{R}, k=1,2, \ldots, n$, $j=1,2, \ldots, m, \sigma-n \times n$ dimensional matrix with constant elements, $h_{i} \in \mathbf{R}^{\mathbf{m}}, i=0,1,2, \ldots, M$ - constant delays in control satisfying the following inequalities:

$$
0=h_{0}<h_{1}<\ldots<h_{i}<\ldots<h_{M-1}<h_{M} .
$$

In the paper, constraints put directly on control values will be consider. Let $S \subset \mathbf{R}^{n}$ be any non-empty set.

\section{Stochastic relative controllability}

3.1. Basic definitions. In this section we define various types of stochastic relative controllability with constrained values of control for the dynamical system (1) in the time interval $\langle 0, T\rangle$.

Definition 1. The dynamical system (1) is said to be stochastically relatively $U$-controllable in the time interval $\langle 0, T\rangle$ from the complete state $z(0) \in \mathbf{R}^{n} \times L^{2}\left(\left\langle v_{M}(0), 0\right\rangle, U\right)$ into the set $S \subset \mathbf{R}^{n}$ if for every vector $\widetilde{x} \in S$, there exist an admissible control $\widetilde{u} \in L_{F}^{2}(\langle 0, T\rangle, U)$ such that the corresponding trajectory $x(t, z(0), \widetilde{u})$ of the dynamical system (1) satisfies $x(T, z(0), \widetilde{u})=\widetilde{x}$.

Definition 2. The dynamical system (1) is said to be (globally) stochastically relatively $U$-controllable in the time interval $\langle 0, T\rangle$ into the set $S$ if it is stochastically relatively $U$ controllable in $\langle 0, T\rangle$ into $S$ for every initial complete state $z(0) \in \mathbf{R}^{n} \times L_{F}^{2}\left(\left\langle v_{M}(0), 0\right\rangle, U\right)$.

Definition 3. The dynamical system (1) is said to be (globally) stochastically relatively $U$-controllable into the set $S$ if for every initial complete state $z(0) \in \mathbf{R}^{n} \times L_{F}^{2}\left(\left[v_{M}(0), 0\right], U\right)$, there exists $T \in[0, \infty)$ such that (1) is relatively $U$ controllable in $[0, T]$ into $S$.

If $S=\mathbf{R}^{n}$, then we talk about (global) stochastically relative $U$-controllability in $\langle 0, T\rangle$. When $S=\{0\}$, we talk about stochastically relative null $U$-controllability in $\langle 0, T\rangle$ from the complete state $z(0)$, and (global) stochastically relative null $U$-controllability in $\langle 0, T\rangle$.

Assume that $S$ is a linear variety in $\mathbf{R}^{n}$ of the form

$$
S=\left\{x \in \mathbf{R}^{n}: \mathbf{L} x=c\right\},
$$

where $\mathbf{L}$ is a known $p \times n$ matrix of rank $p$ and $c \in \mathbf{R}^{p}$ is a given vector. If $\mathbf{L}=\mathbf{I}_{n}(n \times n$ unit matrix $)$ and $c=0$, we get $S=\{0\}$. 
On constrained stochastic controllability of dynamical systems...

The attainable set from the initial complete state $z(0)$ at time $t \in\langle 0, T\rangle$ for the dynamical system (1) has the form

$$
\begin{gathered}
K_{U}(\langle 0, t\rangle, z(0))=\left\{x \in \mathbf{R}^{n}:\right. \\
x=\Phi(t, 0) x_{0}+\int_{0}^{t} \Phi(t, s) \sum_{i=0}^{M} B_{i}(s) u\left(v_{i}(s)\right) d s \\
\left.+\int_{0}^{t} \Phi(t, s) \sigma(s) d w(s), u \in L_{F}^{2}(\langle 0, t\rangle, U)\right\} .
\end{gathered}
$$

3.2. Constrained controllability criteria. In order to formulate criteria of controllability with constrained controls for the stochastic system (1), with the assumption that the final set takes the form (4), let us introduce a scalar function $J: \mathbf{R}^{n} \times \mathbf{R} \times \mathbf{R}^{p} \rightarrow \mathbf{R}$. The function is related to the attainable set $K_{U}(\langle 0, t\rangle, z(0))$ of the system (1) and defined by

$$
\begin{gathered}
J(z(0), t, a)=v^{T} \mathbf{L}\left(\Phi(t, 0) x_{0}+\int_{0}^{t} \Phi(t, s) \sigma(s) d w(s)\right) \\
+\int_{0}^{t} \sup \left\{a^{T} \mathbf{L} \Phi(t, s) \sum_{i=0}^{M} B_{i}(s) u\left(v_{i}(s)\right):\right. \\
\left.u \in L_{F}^{2}(\langle 0, t\rangle, U)\right\} d s-a^{T} c,
\end{gathered}
$$

where $a \in \mathbf{R}^{p}$ is any vector, is called the supporting function of the attainable set. Its application for dynamical systems without delays can be found in [19].

Using the absolute continuity of $v_{i}$ and exploiting their inverses $r_{i}:\left\langle v_{i}(0), v_{i}(T)\right\rangle \rightarrow\langle 0, T\rangle, i=0,1,2, \ldots, M$, we can write the solution of (1) in the following form:

$$
\begin{gathered}
x(t, z(0), u)=\Phi(t, 0) x_{0}+\int_{0}^{t} \Phi(t, s) \sigma(s) d w(s) \\
\quad+\sum_{i=0}^{M} \int_{v_{i}(0)}^{v_{i}(T)} \Phi\left(t, r_{i}(s)\right) B_{i}\left(r_{i}(s)\right) \dot{r}_{i}(s) u(s) d s
\end{gathered}
$$

where

$$
\dot{r}_{i}(s)=\frac{d}{d s} r_{i}(s)
$$

Let us fix a final time $T>0$. Without loss of generality, for simplicity of notation, we may assume that $v_{k}(T)=0$ for some $k \geq M$. If such a $k$ does not exist, then we introduce an additional delay $h_{k}$ with control matrix $B_{k}(t)=0$. Then the solution (2) of the dynamical system (1) has, at time $T$, the form [1, 3]

$$
\begin{aligned}
& x(T, z(0), u)=\Phi(T, 0) x_{0}+\int_{0}^{T} \Phi(t, s) \sigma(s) d w(s) \\
& +\sum_{i=0}^{k} \int_{v_{i}(0)}^{0} \Phi\left(T, r_{i}(s)\right) B_{i}\left(r_{i}(s)\right) \dot{r}_{i}(s) u_{0}(s) d s \\
& +\sum_{i=k+1}^{M} \int_{v_{i}(0)}^{v_{i}(T)} \Phi\left(T, r_{i}(s)\right) B_{i}\left(r_{i}(s)\right) \dot{r}_{i}(s) u_{0}(s) d s \\
& +\sum_{i=0}^{k} \int_{v_{i}(T)} \Phi\left(T, r_{i}(s)\right) B_{i}\left(r_{i}(s)\right) \dot{r}_{i}(s) u(s) d s .
\end{aligned}
$$

The first four terms on the right hand side of the above equation depend on $z(0)$ and $\sigma(t)$ in the interval $\langle 0, T\rangle$, but not on $u$. To simplify the notation we set

$$
\begin{aligned}
& q(z(0), \sigma(t))=x_{0}+\int_{0}^{T} \Phi(0, s) \sigma(s) d w(s) \\
& +\sum_{i=0}^{k} \int_{v_{i}(0)}^{0} \Phi\left(0, r_{i}(s)\right) B_{i}\left(r_{i}(s)\right) \dot{r}_{i}(s) u_{0}(s) d s \\
& +\sum_{i=k+1}^{M} \int_{v_{i}(0)}^{v_{i}(T)} \Phi\left(0, r_{i}(s)\right) B_{i}\left(r_{i}(s)\right) \dot{r}_{i}(s) u_{t_{0}}(s) d s
\end{aligned}
$$

and for $t \in\left\langle v_{i+1}(T), v_{i}(T)\right), i=0,1,2, \ldots, k-1$,

$$
B_{T}(t)=\sum_{j=0}^{i} \Phi\left(0, r_{j}(t)\right) B_{j}\left(r_{j}(t)\right) \dot{r}_{j}(t) .
$$

Let us notice that the integral

$$
\int_{0}^{T} \Phi(0, s) \sigma(s) d w(s)
$$

can be defined pathwise (separately for each $\omega \in \Omega$ ) as the Riemann-Stieltjes integral if the paths have finite variation [20]. This is secured by our assumptions.

Resuming the above transformations and introduced notations we can formulate the following theorem, basing on the result presented in [1].

\section{Lemma 1. Let}

$$
\dot{y}(t)=A(t) y(t)+B_{T}(t) u(t), \quad t \in\langle 0, T\rangle,
$$

be a linear, time-dependent dynamical system without delays in control. Then

$$
x(t, z(0), u)=y(t, q(z(0), \sigma(t)), u), \quad t \in\langle 0, T\rangle .
$$

By Lemma 1, the stochastic relative controllability in $\langle 0, T\rangle$ of the dynamical system (1) and the controllability in $\langle 0, T\rangle$ of the dynamical system without delays in control (8) are equivalent. 
B. Sikora and J. Klamka

For the system (8) the supporting function $J$, at $t=T$, takes the form

$$
\begin{gathered}
J(q(z(0), \sigma(t)), T, a)=a^{T} \mathbf{L} \Phi(T, 0) q(z(0), \sigma(t)) \\
+\int_{0}^{T} \sup \left\{a^{T} \mathbf{L} \Phi(T, s) B_{T}(s) u(s):\right. \\
\left.u \in L_{F}^{2}(\langle 0, T\rangle, U)\right\} d s-a^{T} c .
\end{gathered}
$$

Here $B_{T}(t)$ is an matrix of square integrable functions in $\langle 0, T\rangle$, so the integral in the above formula is properly defined.

Now we can formulate new criteria of stochastic relative controllability for the system (1).

Theorem 1. Let $U$ be a compact set and $E \subset \mathbf{R}^{p}$ be any set containing 0 as an interior point. Then the dynamical system (1) with delays in control is stochastically relatively $U$-controllable from the complete state $z(0) \in$ $\mathbf{R}^{n} \times L_{F}^{2}\left(\left\langle v_{M}(0), 0\right\rangle, U\right)$ into the set $S$ of the form (4) if and only if for some $T \in[0, \infty)$,

$$
\min \{J(q(z(0), \sigma(t)), T, a): a \in E\}=0
$$

or, equivalently,

$$
J(q(z(0), \sigma(t)), T, a) \geq 0 \text { for every } a \in E,
$$

where $J(q(z(0), \sigma(t)), T, a)$ is defined by (9).

Proof: The idea of the proof is similar to that for linear continuous systems with time-dependent multiple delays in control presented in [13]. By Lemma 1 the attainable set $K_{U}$ for (8) takes the form

$$
\begin{gathered}
K_{U}([0, T], q(z(0), \sigma(t))) \\
=\left\{x \in \mathbf{R}^{n}: x=\Phi(T, t) q(z(0), \sigma(t))\right. \\
\left.+\int_{0}^{T} \Phi(T, s) B_{T}(s) u(s) d s, u \in L_{F}^{2}(\langle 0, T\rangle, U)\right\} .
\end{gathered}
$$

We shall show that this set is convex and compact. To prove its compactness, we will show that every sequence of points $x_{1}(T), x_{2}(T), \ldots, x_{k}(T), \ldots$ from $K_{U}(\langle 0, T\rangle, q(z(0), \sigma(t)))$ has a subsequence convergent to some $\bar{x}(T) \in K_{U}(\langle 0, T\rangle, q(z(0), \sigma(t)))$. Since the set $L_{F}^{2}(\langle 0, T\rangle, U)$ of admissible controls is weakly compact in $L_{F}^{2}\left(\langle 0, T\rangle, \mathbf{R}^{m}\right)$ (see [21]), there exists a subsequence of controls $u_{k_{i}} \in L_{F}^{2}(\langle 0, T\rangle, U)$ weakly convergent to some control $\bar{u}$ such that

$$
\lim _{k_{i} \rightarrow \infty} \int_{0}^{T} \Phi(T, s) B_{T}(s) u_{k_{i}}(s) d s=\int_{0}^{T} \Phi(T, s) B_{T}(s) \bar{u}(s) d s .
$$

Let $\bar{x}(t)$ be the solution corresponding to $\bar{u}(t)$. Then in $\langle 0, T\rangle$ we have

$$
\begin{gathered}
\bar{x}(t)=\Phi(t, 0) q(z(0), \sigma(t))) \\
+\int_{0}^{t} \Phi(t, s) B_{T}(s) \bar{u}(s) d s=\lim _{k_{i} \rightarrow \infty} x_{k_{i}}(t) .
\end{gathered}
$$

Therefore

$$
\lim _{k_{i} \rightarrow \infty} x_{k_{i}}\left(t_{1}\right)=\bar{x}(T) \in K_{U}(\langle 0, T\rangle, q(z(0), \sigma(t))) .
$$

The convexity of attainable set is proved in [22] and [23]. It follows that the set $\widetilde{K}_{U}(\langle 0, T\rangle, q(z(0), \sigma(t)))$ of the form

$$
\begin{gathered}
\widetilde{K}_{U}(\langle 0, T\rangle, q(z(0), \sigma(t))) \\
=\left\{y \in \mathbf{R}^{p}: y=\mathbf{L} x, x \in K_{U}(\langle 0, T\rangle, q(z(0), \sigma(t)))\right\}
\end{gathered}
$$

is also convex and compact. An initial complete state $x_{0}$ can be steered to the set $S$ in time $T>0$ if and only if the vector $c$ and the set $\widetilde{K}_{U}(\langle 0, T\rangle, q(z(0), \sigma(t)))$ cannot be strictly separated by a hyperlane, that is, if

$$
a^{T} c \leq \sup \left\{a^{T} \widetilde{x}: \widetilde{x} \in \widetilde{K}_{U}(\langle 0, T\rangle, q(z(0), \sigma(t)))\right\}
$$

for all vectors $a \in \mathbf{R}^{p}$. This follows from a theorem about separating convex sets [24].

The above inequality is equivalent to:

$$
\begin{gathered}
a^{T} \mathbf{L} \Phi(T, 0) q(z(0), \sigma(t)) \\
+\sup \left\{\int_{0}^{T} a^{T} \mathbf{L} \Phi(T, s) B_{T}(s) u(s) d s:\right. \\
\left.u \in L_{F}^{2}(\langle 0, T\rangle, U)\right\}-a^{T} c \geq 0 .
\end{gathered}
$$

Interchanging integration and taking supremum we conclude that $c \in \widetilde{K}_{U}(\langle 0, T\rangle, q(z(0), \sigma(t)))$ if and only if $J(q(z(0), \sigma(t)), T, a) \geq 0$ for all $a \in \mathbf{R}^{p}$.

Moreover, we can show that

$$
\begin{gathered}
k J(q(z(0), \sigma(t)), T, a)=J(q(z(0), \sigma(t)), T, k a) \\
\text { for every } \quad k \geq 0 .
\end{gathered}
$$

Therefore, restricting to vectors $a \in E$, we obtain the assertion of the theorem.

Theorem 2. Let $U \subset \mathbf{R}^{m}$ be an compact set and $E \subset \mathbf{R}^{n}$ be any set containing 0 as an interior point. Then the dynamical system (1) is stochastically relatively null $U$-controllable from the complete state $z(0) \in \mathbf{R}^{n} \times L_{F}^{2}\left(\left\langle v_{M}(0), 0\right\rangle, U\right)$ if and only if for some $T \in\langle 0, \infty)$

$$
\min \{J(q(z(0), \sigma(t)), T, a): a \in E\}=0
$$

or, equivalently,

$$
J(q(z(0), \sigma(t)), T, a) \geq 0 \text { for every } a \in E .
$$

Proof. This follows directly from Theorem 1 for $S=\{0\}$, i.e. for $\mathbf{L}=\mathbf{I}_{\mathbf{n}}$ and $c=0$. Then $E$ is a subset of $\mathbf{R}^{n}$.

Obviously, both the above theorems hold also for the stationary system (3). For the system (3) the transition matrix $\Phi(0, t)$ equals $e^{A t}$, and the attainable set from the initial complete state $z(0)$ at time $t \in\langle 0, T\rangle$ takes the form: 
On constrained stochastic controllability of dynamical systems..

$$
\begin{aligned}
& K_{U}(\langle 0, t\rangle, z(0))=\left\{x \in \mathbf{R}^{n}: x=e^{A t} x_{0}\right. \\
& \left.+\int_{0}^{t} e^{A(t-s)} \sum_{i=0}^{M} B_{i}(s) u\left(s-h_{i}\right)\right) d s \\
& \left.+\int_{0}^{t} e^{A(t-s)} \sigma d w(s), u \in L_{F}^{2}(\langle 0, t\rangle, U)\right\} .
\end{aligned}
$$

Therefore, the corresponding supporting function $J$, at $t=T$, is described by the formula

$$
\begin{gathered}
J(q(z(0), \sigma), T, a)=a^{T} \mathbf{L} e^{A T} q(z(0), \sigma) \\
+\int_{0}^{T} \sup \left\{a^{T} \mathbf{L} e^{A(T-s)} B_{T}(s) u(s):\right. \\
\left.u \in L_{F}^{2}(\langle 0, T\rangle, U)\right\} d s-a^{T} c,
\end{gathered}
$$

where

$$
\begin{gathered}
q(z(0), \sigma)=x_{0}+\int_{0}^{T} e^{A s} \sigma d w(s) \\
+\sum_{i=0}^{k} \int_{v_{i}(0)}^{0} e^{A\left(r_{i}(s)\right)} B_{i}\left(r_{i}(s)\right) \dot{r}_{i}(s) u_{0}(s) d s \\
+\sum_{i=k+1}^{M} \int_{v_{i}(0)}^{v_{i}(T)} e^{A\left(r_{i}(s)\right)} B_{i}\left(r_{i}(s)\right) \dot{r}_{i}(s) u_{t_{0}}(s) d s
\end{gathered}
$$

and for $t \in\left\langle v_{i+1}(T), v_{i}(T)\right), i=0,1,2, \ldots, k-1$

$$
B_{T}(t)=\sum_{j=0}^{i} e^{A\left(r_{j}(s)\right)} B_{j}\left(r_{j}(t)\right) \dot{r}_{j}(t),
$$

for $v_{i}(t)=t-h_{i}$ and $r_{i}(t)=v_{i}^{-1}(t)$.

\section{Conclusions}

The linear stochastic dynamical systems with multiple, timedependent delays in control and constraints on control values have been analyzed in the paper. Various types of stochastic relative controllability with constrained values of control for the dynamical system (1) have been defined. The criteria of stochastic relative controllability with constrained control have been established and proved. The results obtained in this paper extend the results for systems with constrained delays in control [13] and stochastic systems with delays in control [7].

Further works of the authors will focus on fractional systems with delays [25] and positive fractional systems [26].

Acknowledgements. This work was partially supported by The National Centre for Research and Development under grant no. OR 00013212.

\section{REFERENCES}

[1] J. Klamka, Controllability of Dynamical Systems, Kluwer, Dordrecht, 1991.

[2] J. Klamka, "Stochastic controllability of linear systems with state delays", Int. J. Appl. Math. Comput. Sci. 17 (1), 5-13 (2007).
[3] J. Klamka, "Stochastic controllability of systems variable delay in control”, Bull. Pol. Ac.: Tech. 56 (4), 279-284 (2008).

[4] J. Klamka, "Controllability of dynamical systems - A survey", Archives Control Science 2 (3/4), 281-307 (1993).

[5] B. Sikora, "On controllability and minimum energy control of linear positive systems with delays", Archives of Control Science 13 (4), 431-444 (2003).

[6] J. Klamka, L. Socha, "Some remarks about stochastic controllability for delayed linear systems", Int. J. Control 32 (3), 561-566 (1980).

[7] J. Klamka, "Stochastic controllability of systems with multiple delays in control", Int. J. Appl. Math. Comput. Sci. 19 (1), 39-47 (2009).

[8] J. Zabczyk, "Controllability of stochastic linear systems", Systems and Control Letters 1 (1), 25-31 (1991).

[9] N.I. Mahmudov, "Controllability of linear stochastic systems", IEEE Trans. Automatic Control 46 (5), 724-731 (2001).

[10] N.I. Mahmudov, "Controllability of linear stochastic systems in Hilbert spaces", J. Mathematical Analysis and Applications 259, 64-82 (2001).

[11] N.I. Mahmudov and A. Denker, "On controllability of linear stochastic systems", Int. J. Control 73 (2), 144-151 (2000).

[12] B. Sikora, "On the constrained controllability of dynamical systems with multiple delays in the state", Int. J. Appl. Math. Comput. Sci. 13 (4), 469-479 (2003).

[13] B. Sikora, "On constrained controllability of dynamical systems with multiple delays in control", Appl. Math. 32 (1), 87 101 (2005).

[14] T. Kaczorek, "Observability of the linear stationary systems with the stochastic inputs", Bull. Pol. Ac.: Tech. 18 (2), 163 168 (1970).

[15] T. Kaczorek, "Observability of the multi-dimensional linear stationary systems with the stochastic inputs", Bull. Pol. Ac.: Tech. 18 (4), 327-331 (1970).

[16] T. Kaczorek, "Observability of the linear nonstationary systems with stochastic inputs", Proc. Fourth Colloquium on Microwave Communication 1 (16), (1-10) 1970.

[17] L. C. Evans, "An introduction to stochastic differential equations", version 1.2, Course Notes, Department of Mathematics 1, CD-ROM (2003).

[18] B. Øksendal, Stochastic Differential Equations. An Introduction with Applications, Sixth Edition, Springer-Verlag, Berlin, 2003.

[19] W. Schmitendorf and B. Barmish, "Null controllability of linear systems with constrained controls", SIAM J. Control Optim. 18, 327-345 (1980).

[20] Z. Brzeźniak and T. Zastawniak, Basic Stochastic Processes: A Course Through Exercise, Springer, London, 2006.

[21] E. Lee and L. Markus, "Bases of optimal control theory", Nauka 1, CD-ROM, 1972, (in Russian).

[22] L.W. Neustadt, "The existence of optimal controls in the absence of convexity conditions", J. Math. Anal. Appl. 7, 110117 (1963).

[23] C. Olech, "Extremal solutions of a control system", J. Differential Equations 2, 74-101 (1966).

[24] T. Kaczorek, Automatic regulation systems theory, WNT, Warszawa, 1977, (in Polish).

[25] Z. Zaczkiewicz, "Representation of solutions for fractional differental - algebraic systems with delays", Bull. Pol. Ac.: Tech. 58 (4), 607-612 (2010).

[26] T. Kaczorek, "Positive fractional 2D continuous - discrete linear systems", Bull. Pol. Ac.: Tech. 59 (4), 575-579 (2011). 